\title{
The blood perfusion and NADH/FAD content combined analysis in patients with diabetes foot
}

\author{
Victor V. Dremin*a ${ }^{* a}$, Viktor V. Sidorov ${ }^{\mathrm{b}}$, Alexander I. Krupatkin ${ }^{\mathrm{c}}$, Gagik R. Galstyan ${ }^{\mathrm{d}}$, Irina N. \\ Novikova ${ }^{a}$, Angelina I. Zherebtsova ${ }^{a}$, Evgeny A. Zherebtsov ${ }^{\mathrm{a}}$, Andrey V. Dunaev ${ }^{\mathrm{a}}$, Zera N. \\ Abdulvapova $^{\mathrm{d}}$, Karina S. Litvinova ${ }^{\mathrm{e}}$, Ilya E. Rafailov ${ }^{\mathrm{f}}$, Sergei G. Sokolovski ${ }^{\mathrm{e}}$, Edik U. Rafailov ${ }^{\mathrm{e}}$ \\ ${ }^{a}$ Biomedical Photonics Instrumentation Group, Scientific-Educational Center "Biomedical \\ Engineering", State University - Education-Science-Production Complex, Oryol, 302020, Russia; \\ ${ }^{b}$ SPE "LAZMA" Ltd., Moscow, 125252, Russia; 'Priorov Central Research Institute of \\ Traumatology and Orthopaedics, Moscow, 127299, Russia; ${ }^{\mathrm{d}}$ Endocrinology Research Center of the \\ Ministry of Health of the Russian Federation, Moscow, 117036, Russia; ${ }^{\mathrm{e}}$ Optoelectronics and \\ Biomedical Photonics Group, Aston Institute of Photonic Technologies, Aston University, Aston \\ Triangle, Birmingham, B4 7ET, UK; ${ }^{\mathrm{f}}$ School of Engineering and Applied Sciences, Aston Institute \\ of Photonic Technologies, Aston University, Aston Triangle, Birmingham, B4 7ET, UK
}

\begin{abstract}
Skin blood microcirculation and the metabolism activity of tissue were examined on the patients with type 2 diabetes. Laser Doppler flowmetry (LDF) with $1064 \mathrm{~nm}$ laser light source and fluorescence spectroscopy (FS) with excitation light of $365 \mathrm{~nm}$ and $450 \mathrm{~nm}$ have been used to monitor the blood perfusion and the content of coenzymes NADH and FAD.
\end{abstract}

Concluding, the proposed combined LDF and tissue FS approach allows to identify the significant violations in the blood microcirculation and metabolic activity for type 2 diabetes patients.

Keywords: diabetic foot syndrome, microcirculation, redox ratio, fluorescence spectroscopy, laser Doppler flowmetry.

\section{INTRODUCTION}

According to the International Diabetes Federation (IDF) report for 2015, there are 415 million diabetic patients with this figure projected to grow to 642 million worldwide by $2040^{1}$. The diagnosis of vascular complications and monitoring of treatment effectiveness of diabetes is one of the most acute in modern healthcare.

Novel compact lasers and laser systems can significantly increase reliability of the diagnostic devices for general practitioners and hospital clinicians and consequently improve people's health and life expectancy. This promising direction in optical diagnostics development is to provide scientific and instrumental ideas and solutions for in situ analysis of body physiological parameters in normality/pathology.

Beside wide applications of these methods in modern medicine, it can also be used for early foot vascular disorder diagnostics in patients with type 2 diabetes.

Currently, the gold standards of diabetic foot disorder detection in surgery or hospitals are predominantly visual inspection and ultrasound Doppler or duplex scanning of arteries. These methods are capable of determining the level of trophic disorders, specificity of the haemodynamics and blood flows. However, at the distal side of feet with microcirculatory disorders, ultrasound Doppler is not efficient enough ${ }^{2}$. Other method like radio-contrast angiography have significant limitation during regular patient control: invasiveness, contrast agent toxicity and prolong exposure of the patient to radiation ${ }^{3,4}$. Comparably, the new approach of transcutaneous oximetry allows one to assess the saturated tissue oxygen. Beside non-invasiveness, this method is time-consuming and demonstrates low accuracy due to different clinical factors ${ }^{5,6}$. Another widely used approach is thermal imaging which reveals thermoasymmetry zones on the foot surface, however, it demonstrates low accuracy in defining the boundaries of the trophic lesions ${ }^{7,8}$.

*dremin_viktor@mail.ru; phone 74862 419876; bmecenter.ru/en

Advanced Biomedical and Clinical Diagnostic and Surgical Guidance Systems XIV, edited by Tuan Vo-Dinh, Anita Mahadevan-Jansen, Warren S. Grundfest, Proc. of SPIE Vol. 9698, 969810

(c) 2016 SPIE · CCC code: 1605-7422/16/\$18 - doi: 10.1117/12.2212758 
In recent years, optical methods of microvasculature diagnosis have been actively developed, in particular laser Doppler flowmetry (LDF) $)^{9,10}$, as well as methods based on the analysis of skin temperature fluctuations ${ }^{11}$. LDF is the most widely used method for monitoring the microcirculation, and assessing its level in tissue. However, the technique of laser Doppler flowmetry has some limitations related to an inadequately developed methodological base.

This study aims to improve the diagnosis of diabetic foot by combining the most commonly used method to date with LDF and fluorescence spectroscopy (FS) ${ }^{12-14}$. This integration will provide a comprehensive analysis of the changes in the tissues of patients with diabetes, potentially increase the informative value and reliability of the diagnostic results.

Metabolic activity is an important parameter of tissue viability. One estimate of metabolic activity is the ratio of coenzymes NADH and FAD, determined by the intensity of their endogenous fluorescence. Changes in the mitochondrial respiratory chain activity is a known consequence of disturbances in the functioning of the cells during different pathologies, especially at diabetes. It should assumed that the changes in the accumulation of both coenzymes NADH (the reduced form) and FAD (oxidized form) can most likely be acquired. Detection of such changes by fluorescence spectroscopy is one of the most promising directions of in vivo diagnosis ${ }^{15-21}$.

The aim of this study was an attempt to experimentally examine the relationship between blood flow and oxidative metabolism and to analyse the potential parameter co-registration of blood flow and skin coenzyme fluorescence to diagnose disorders of the lower extremities in patients with type 2 diabetes.

\section{MATERIAL AND METHODS}

In complex experimental studies the tissue blood perfusion and the content of coenzyme NADH and FAD were assessed by LDF and FS respectively. Registered perfusion parameters and the amplitude of the coenzymes' fluorescence were simultaneously evaluated using a "LAZMA MC" system (SPE "LAZMA" Ltd, Russia) in in the same tissue volume. The Doppler channel built on the basis of the laser module used a wavelength of $1064 \mathrm{~nm}$. For fluorescence excitation of $\mathrm{NADH}$ and FAD, $365 \mathrm{~nm}$ and $450 \mathrm{~nm}$ sources of radiation were used respectively. Delivery of probing radiation and receiving back reflected secondary radiation from the tissue were realised by a multiple optical fibre probe (Fig. 1).

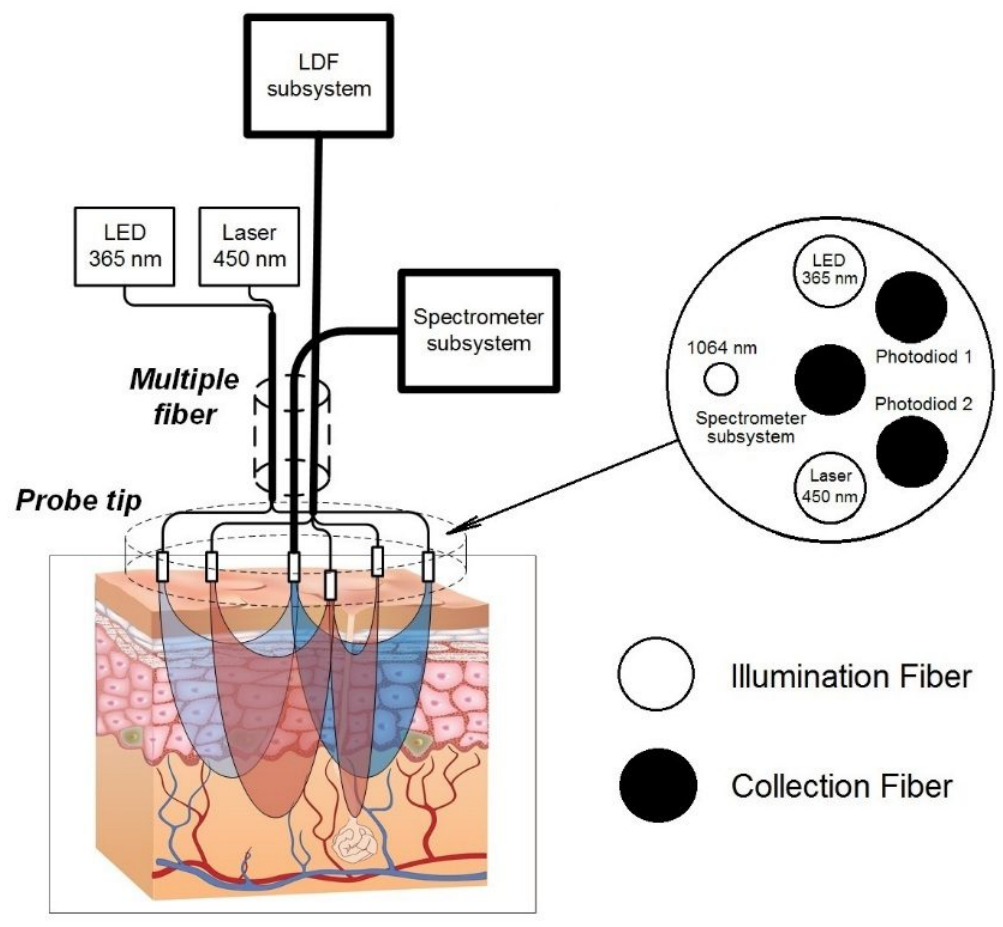

Figure 1. The general scheme of "LAZMA MC" system. 
A specialised software was developed to work with the system. This allowed for real-time control of the course of the experiment and analysis of recorded parameters, control unit and realising functional tests - thermal- and electrostimulation. Fig.2 shows an example of the working window of the software: top panel - the registered fluorescence spectrum at UV radiation; bottom panel - registered LDF-grams during the heating of samples with different modes.

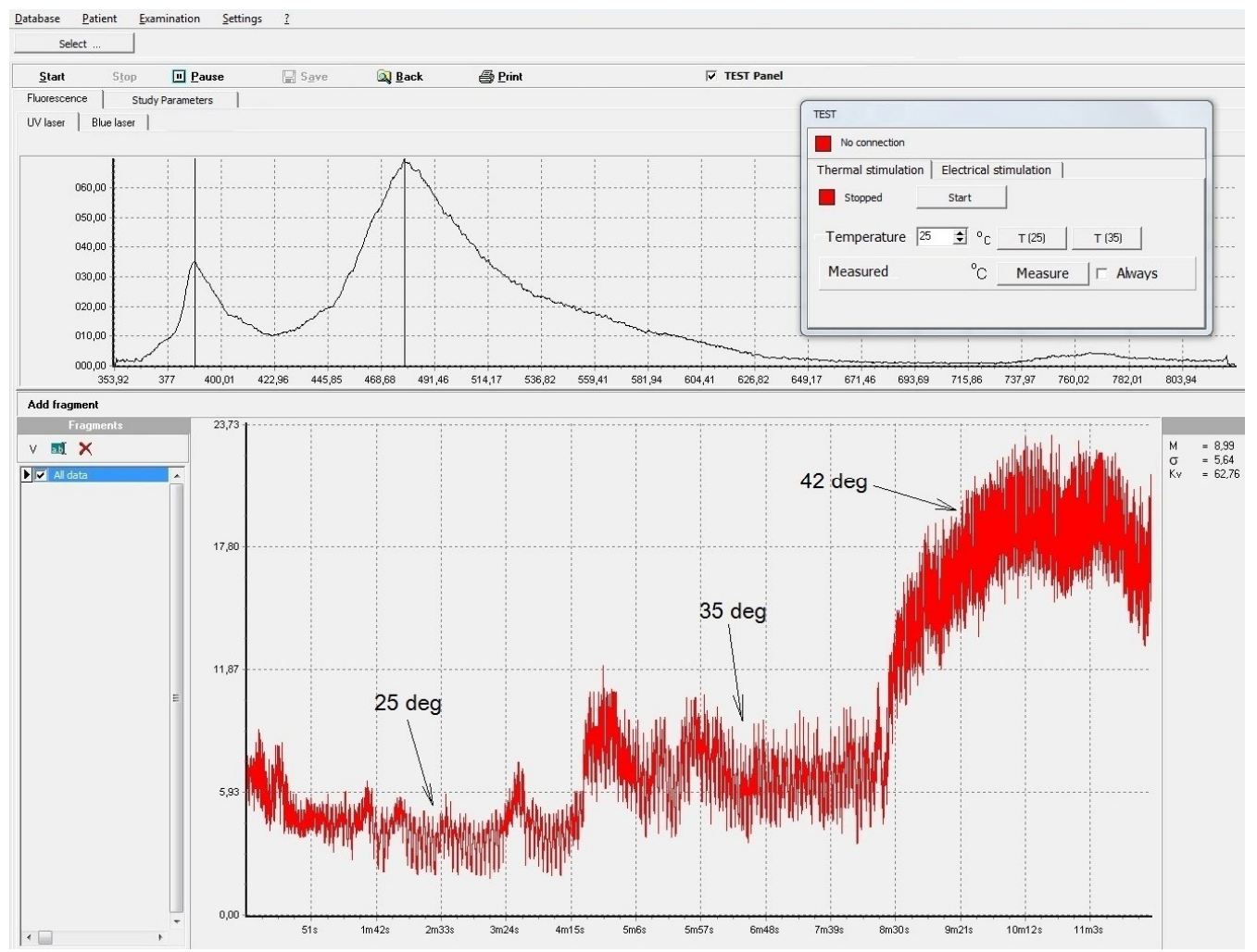

Figure 2. An example of the working window of the software for "LAZMA MC" with an actual records of the foot skin fluorescence and blood perfusion (lower section).

The experimental study included two stages. The first stage was conducted in 15 patients with type 2 diabetes aged $63 \pm 7$ years (at the Endocrinology Department "Oryol Regional Hospital"). The control group consisted of 15 apparently healthy volunteers (mean age $53 \pm 5$ years). Before investigations participants signed informed consent indicating their willingness to participate in the study.

Background records $(5 \mathrm{~min})$ with 2 points of the top side of the foot $\left(1^{\text {st }}\right.$ top point on the medial line of the foot, $2^{\text {nd }}$ lowest point on the line between the $1^{\text {st }}$ and $2^{\text {nd }}$ metatarsal bones) on both legs (arrangement of the fibres and the foot of patients are presented in Fig. 3a) were obtained. The "LAZMA MC" system simultaneously recorded a pair of spectra during the perfusion recording, with an interval of 30 seconds. Thus, a total of 10 spectra pairs were recorded during the diagnostic procedure. A total of 75 patients aged $53 \pm 13$ years were involved at the second stage of the experimental study. Local cold $\left(25^{\circ} \mathrm{C}\right)$ and the thermal probe $\left(35^{\circ} \mathrm{C}\right.$ and $\left.42^{\circ} \mathrm{C}\right)$ was applied at this stage using the "LAZMA-TEST" system (SPE "LAZMA" Ltd, Russia) (Fig. 3b). The "LAZMA-TEST" system is intended for performing a selective functional loan on the blood microcirculatory system during investigations. The system implements a: temperature test (from 5 to $50^{\circ} \mathrm{C}$ ), skin temperature measurement and electrostimulation test. 


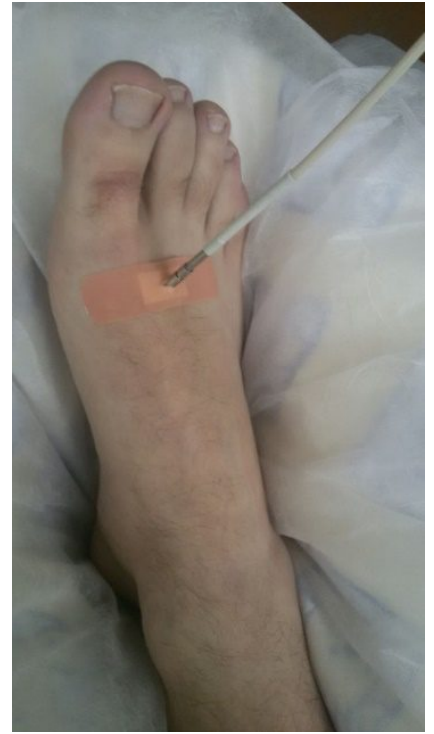

a)

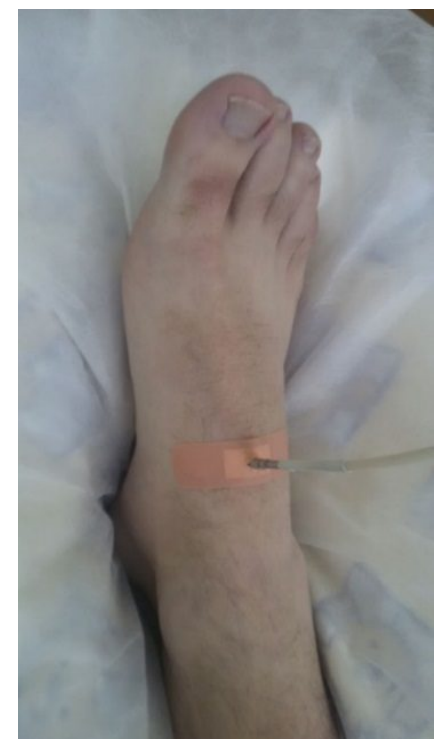

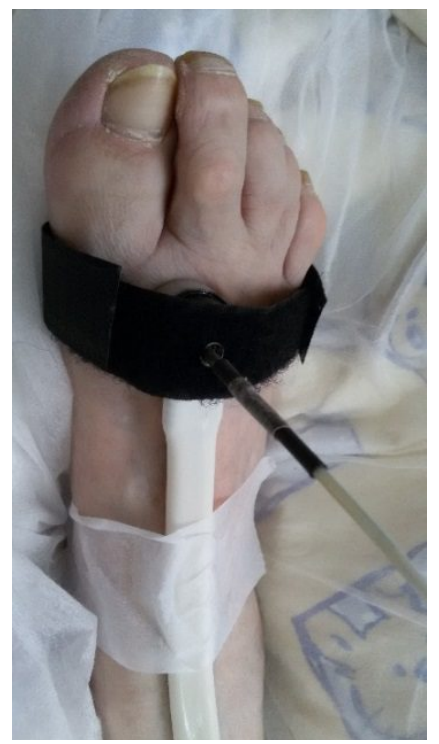

b)

Figure 3. The localisation of the fibre optic probe on the diabetic foot (a) and the same with Peltier thermal-pad (b) during the measurements.

A typical example of registered fluorescence spectra and perfusion pairs using "LAZMA MC" system is presented in Fig. 4.

Multiple parameters were analysed. The amplitude of the fluorescence $\mathrm{I}_{\mathrm{NADH}}$ и $\mathrm{I}_{\mathrm{FAD}}$, normalized to the intensity of the excitation radiation $\mathrm{I}_{365}$ and $\mathrm{I}_{450}$ reflected back respectively, redox ratio [Redox], the average blood tissue perfusion $\mathrm{I}_{\mathrm{m}}$, as well as the proposed complex parameter, characterising the intensity of metabolic processes - metabolic rate (MR):

$$
\begin{gathered}
{[\text { Redox }]=\frac{I_{\mathrm{NADH}}}{\mathrm{I}_{\mathrm{FAD}}} \cdot \frac{\mathrm{I}_{450}}{\mathrm{I}_{365}},} \\
\mathrm{MR}=\frac{[\text { Redox }]}{\mathrm{I}_{\mathrm{m}}} .
\end{gathered}
$$

For each 5 minute study, all indicators were averaged over 10 measurements.

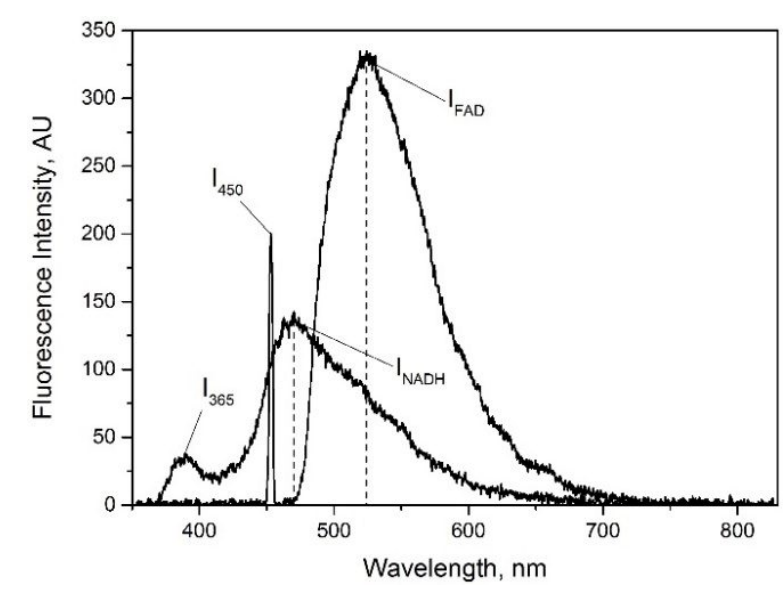

a)

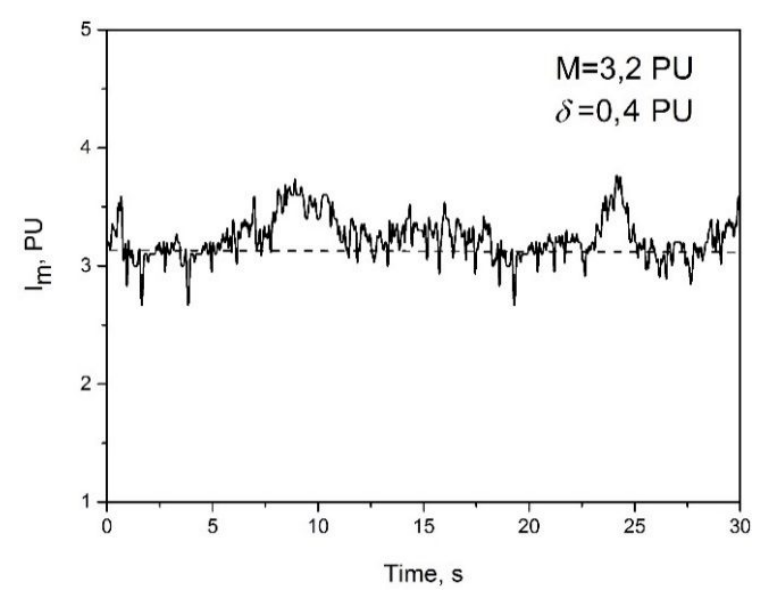

b)

Figure 4. A typical example of fluorescence spectra (a) and blood perfusion record (b) by the "LAZMA MC" system from foot skin of the patients with type 2 diabetes. 
At the second stage of the experimental study perfusion and fluorescence spectra were recorded on the back of the foot (lowest point on the line between the 1st and 2nd metatarsal bones), on both legs (arrangement of fibres with a temperature probe is shown in Fig. 3b). Stages and parameters of the study are presented in Table 1.

Table 1. Method studies using temperature probes.

\begin{tabular}{|l|c|c|}
\hline \multicolumn{1}{|c|}{ Stage } & Temperature, ${ }^{\circ} \mathrm{C}$ & Time, min \\
\hline Basic test & Control of body temperature & 4 \\
\hline Local cooling & 25 & 4 \\
\hline Local heating & 35 & 4 \\
\hline Local heating & 42 & 10 \\
\hline
\end{tabular}

It should be noted that the effect of blood as an absorber in the optical signal during heating of tissue was controlled by the value of the exciting radiation reflected back. It should be further noted that the issues of the blood flow scattering and absorbing effects on the recorded signals in optical biomedical diagnostics have been discussed in a number of publications ${ }^{14,22}$. Accounting for this effect is a prerequisite for the interpretation of diagnostic data.

\section{EXPERIMENTAL RESULTS AND DISCUSSION}

Preliminary data analysis between two topographic anatomical sites from the first stage of the experimental studies did not reveal any statistical differences (by the Mann-Whitney test, $\mathrm{p}<0.05$ ). Thus, we can talk about the good repeatability of measurements in different areas of the foot.

The first phase of the experimental studies have shown that diabetic patients have elevated values of the normalized amplitudes of NADH and FAD. Confirmed by statistically significant differences (Table 2). Increased quantities of $\mathrm{NADH}$ may indicate an increase in the contribution of anaerobic metabolism in diabetic patients.

Table 2. NADH and FAD fluorescence parameter and blood perfusion data from healthy and diabetic foot skin

\begin{tabular}{|l|c|c|}
\hline \multicolumn{1}{|c|}{ Parameter } & $\begin{array}{c}\text { Control } \\
\text { group }\end{array}$ & Diabetes \\
\hline $\begin{array}{l}\text { The normalized amplitude } \\
\text { NADH, AU }\end{array}$ & $3.1 \pm 0.6$ & $3.6 \pm 1.2^{*}$ \\
\hline $\begin{array}{l}\text { The normalized amplitude } \\
\text { FAD, AU }\end{array}$ & $1.1 \pm 0.2$ & $1.8 \pm 0.6^{*}$ \\
\hline Perfusion $\mathrm{I}_{\mathrm{m}}, \mathrm{PU}$ & $6.0 \pm 2.6$ & $6.6 \pm 2.4$ \\
\hline [Redox], AU & $3.1 \pm 0.6$ & $2.1 \pm 0.4^{*}$ \\
\hline Index metabolism MR, AU & $0.6 \pm 0.3$ & $0.4 \pm 0.1^{*}$ \\
\hline
\end{tabular}

$*$ - Confirmed the statistical significance of differences in the Mann-Whitney test $(\mathrm{p}<0.05)$

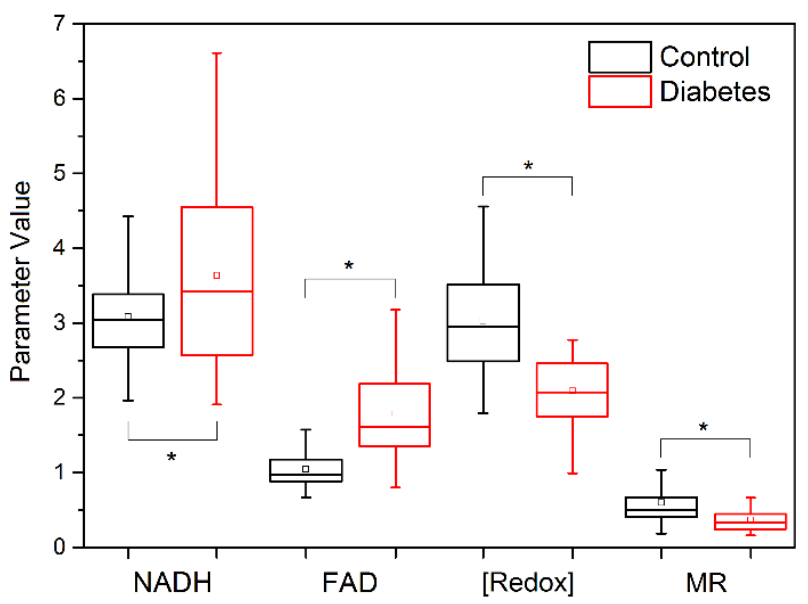

Figure 5. Comparison of parameters between diabetes and healthy groups of patients. In each box, the central line is the median of the group, while the edges are the 25 th and 75 th percentiles.

The complex parameter MR may be used for application in clinical practice as a background recording, which allows to evaluate the biological tissue conditions in normality or pathology. The use of the MR parameter makes it possible to numerically correlate the blood flow and metabolic processes, which are closely related to each other ${ }^{23,24}$. Also this 
parameter allows researchers to correlate the fundamental principle of the tissue metabolism in "oxygen deliveryconsumption" terms as an estimated balance of oxygen and nutrient delivery by blood (perfusion) and its consumption in the cells (NADH and FAD). Thus, the average level of MR for a group of patients in the first stage of the study was $0.4 \pm 0.1$ AU compared to the controls $-0.6 \pm 0.3 \mathrm{AU}$.

The local thermal runaway increase in perfusion was mainly due to two mechanisms. By heating the skin to $34-35{ }^{\circ} \mathrm{C}$ peptidergic sensory nerves are activated. This is due to the activation of thermosensitive vanilloid receptor type 1 sensor (VR-1) fibre ${ }^{25}$. When skin was heated to $42{ }^{\circ} \mathrm{C}$, vasodilation associated with the release of NO from the vascular endothelium is observed ${ }^{26,27}$. During the local heating, these regulatory processes involve both arterioles and capillaries.

Patients with type 2 diabetes exhibit dysfunction of all parts of the microvasculature in the tissue system, including the vascular endothelial and perivascular nerves. Therefore, the thermal test probe can be adopted for the diagnosis of these disorders in diabetes foot. Insufficient increase of skin blood perfusion during the heating to $35^{\circ} \mathrm{C}$ can be implemented as an objective criterion of sensory nerve dysfunction and a component of diabetic neuropathy. The absence of the proper skin blood perfusion response on the heating to $42{ }^{\circ} \mathrm{C}$ demonstrates the deficiency of the endothelium-dependent vasodilation mechanisms ${ }^{9}$ in diabetes. The heating test can be used as an assessment of the adaptive reserves of the "oxygen delivery-consumption" processes in the tissues. At high vasodilation during heating to $42{ }^{\circ} \mathrm{C}$, with correspondent increase in tissue oxygen content, the NADH is intensively oxidised in mitochondria both in healthy and in diabetic patients. Wherein, the dynamics of changes in NADH and FAD is more pronounced for a group of diabetic patients compared to the healthy ones.

\section{CONCLUSION}

These comprehensive experimental investigations reveal the functional state violation of lower extremities in patients with type 2 diabetes. They show the high sensitivity of optical non-invasive methods (laser Doppler flowmetry and fluorescence spectroscopy during their joint application) to detect irregularities in the haemodynamic and metabolic processes of the biological tissue in type 2 diabetes.

Studies of the patients' endocrinological profile show that the proposed original method can be used to diagnose disorders of the microvasculature and metabolism in the lower limbs of patients at the early stages of the disease and clarify the pathogenesis of "diabetic foot".

\section{ACKNOWLEDGMENTS}

This work was supported by the grant of the Ministry of Education and Science, Russian Federation, for State University - Education-Science-Production Complex (state task, GZ №310).

\section{REFERENCES}

[1] [IDF Diabetes Atlas - 7th edition], International Diabetes Federation, 142 (2015).

[2] Ahmadi, M. E., Morrison, W. B., Carrino, J. A., Schweitzer, M. E., Raikin, S. M. and Ledermann, H. P., "Neuropathic arthropathy of the foot with and without superimposed osteomyelitis: MR imaging characteristics," Radiology, 238(2), 622-31 (2006).

[3] Chatha, D. S., Cunningham, P. M. and Schweitzer, M. E., "MR imaging of the diabetic foot: diagnostic challenges," Radiol Clin North Am, 43(4), 747-59 (2005).

[4] Prandini, N., Lazzeri, E., Rossi, B., Erba, P., Parisella, M. G. and Signore, A., "Nuclear medicine imaging of bone infections," Nucl Med Commun, 27(8), 633-44 (2006).

[5] Fife, C. E., Smart, D. R., Sheffield, P. J., Hopf, H. W., Hawkins, G. and Clarke, D., "Transcutaneous oximetry in clinical practice: consensus statements from an expert panel based on evidence," Undersea Hyperb Med, 36(1), 43-53 (2009).

[6] Arsenault, K. A., Al-Otaibi, A., Devereaux, P. J., Thorlund, K., Tittley, J. G. and Whitlock, R. P., "The use of transcutaneous oximetry to predict healing complications of lower limb amputations: a systematic review and meta-analysis," Eur J Vasc Endovasc Surg, 43(3), 329-36 (2012). 
[7] Berendt, A. R. and Lipsky, B., "Is this bone infected or not? Differentiating neuro-osteoarthropathy from osteomyelitis in the diabetic foot," Curr Diab Rep, 4(6), 424-9 (2004).

[8] Boulton, A. J. and Vileikyte, L., "The diabetic foot: the scope of the problem," J Fam Pract, 49(11 Suppl), S3-8 (2000).

[9] Krupatkin, A. I. and Sidorov, V. V., [Laser Doppler flowmetry of blood], Meditcina-Press, Moscow(2005).

[10] Leahy, M. J., De Mul, F. F., Nilsson, G. E. and Maniewski, R., "Principles and practice of the laser-Doppler perfusion technique," Technology and health care: official journal of the European Society for Engineering and Medicine, 7(2-3), 143-162 (1999).

[11] Podtaev, S., Stepanov, R., Smirnova, E. and Loran, E., "Wavelet-analysis of skin temperature oscillations during local heating for revealing endothelial dysfunction," Microvasc Res, 97, 109-14 (2015).

[12] Lakowicz, J. R., [Principles of Fluorescence Spectroscopy], Kluwer Academic Publishers, New York, 954 (2006).

[13] Mycek, M. A. and Pogue, B. W., [Handbook of Biomedical Fluorescence], Taylor \& Francis, (2003).

[14] Dunaev, A. V., Dremin, V. V., Zherebtsov, E. A., Rafailov, I. E., Litvinova, K. S., Palmer, S. G., Stewart, N. A., Sokolovski, S. G. and Rafailov, E. U., "Individual variability analysis of fluorescence parameters measured in skin with different levels of nutritive blood flow," Med Eng Phys, 37(6), 574-83 (2015).

[15] Chance, B. and Williams, G. R., "Respiratory enzymes in oxidative phosphorylation. I. Kinetics of oxygen utilization," J Biol Chem, 217(1), 383-93 (1955).

[16] Chance, B., Williamson, J., Jamieson, D. and Schoener, B., "Properties and kinetics of reduced pyridine nucleotide fluorescence of the isolated and in vivo rat heart," Biochem. J, 341, 357-377 (1965).

[17] Bartolome, F. and Abramov, A. Y., "Measurement of mitochondrial NADH and FAD autofluorescence in live cells," Methods Mol Biol, 1264, 263-70 (2015).

[18] Mayevsky, A. and Chance, B., "Oxidation-reduction states of NADH in vivo: from animals to clinical use," Mitochondrion, 7(5), 330-9 (2007).

[19] Papayan, G., Petrishchev, N. and Galagudza, M., "Autofluorescence spectroscopy for NADH and flavoproteins redox state monitoring in the isolated rat heart subjected to ischemia-reperfusion," Photodiagnosis Photodyn Ther, 11(3), 400-8 (2014).

[20] Aldakkak, M., Stowe, D. F., Lesnefsky, E. J., Heisner, J. S., Chen, Q. and Camara, A. K., "Modulation of mitochondrial bioenergetics in the isolated Guinea pig beating heart by potassium and lidocaine cardioplegia: implications for cardioprotection," J Cardiovasc Pharmacol, 54(4), 298-309 (2009).

[21] An, J., Camara, A. K., Rhodes, S. S., Riess, M. L. and Stowe, D. F., "Warm ischemic preconditioning improves mitochondrial redox balance during and after mild hypothermic ischemia in guinea pig isolated hearts," Am J Physiol Heart Circ Physiol, 288(6), H2620-7 (2005).

[22] Dunaev, A. V., Zherebtsov, E. A., Rogatkin, D. A., Stewart, N. A., Sokolovski, S. G. and Rafailov, E. U., "Substantiation of medical and technical requirements for noninvasive spectrophotometric diagnostic devices," J Biomed Opt, 18(10), 107009 (2013).

[23] Klabunde, R. E., [Cardiovascular physiology concepts], Lippincott Williams \& Wilkins, 148-179 (2012).

[24] Guyton, A. C., [Textbook of medical physiology ], Elsevier Saunders, 195-203 (2006).

[25] Stephens, D. P., Charkoudian, N., Benevento, J. M., Johnson, J. M. and Saumet, J. L., "The influence of topical capsaicin on the local thermal control of skin blood flow in humans," Am J Physiol Regul Integr Comp Physiol, 281(3), R894-901 (2001).

[26] Minson, C. T., Berry, L. T. and Joyner, M. J., "Nitric oxide and neurally mediated regulation of skin blood flow during local heating," J Appl Physiol (1985), 91(4), 1619-26 (2001).

[27] Kellogg, D. L., Jr., Zhao, J. L. and Wu, Y., "Roles of nitric oxide synthase isoforms in cutaneous vasodilation induced by local warming of the skin and whole body heat stress in humans," J Appl Physiol (1985), 107(5), 1438-44 (2009). 